\title{
MEASUREMENT OF THE DEGREE OF SHADING OR CROWN CANOPY DENSITY IN FOREST SITES
}

\author{
By J. G. WRIGHT \\ Dominion Forest Service, Ottawa, Canada.
}

P RIOR to 1941, the only instrument used for measuring the density of crown canopy for forest-fire hazard studies was the Clements Photometer. The light sensitive element in this instrument is a narrow band of sensitized photographic paper (daylight). The band, enclosed in a light proof cylinder, can be rotated to pass under a small window fitted with a shutter. A series of exposures can be made on the band and timed with the stopwatch attached to the instrument.

The method usually followed is to make the first twelve exposures in full sunlight in the open, giving exposure No. 1 one second, No. 2 two seconds and so on, exposure No. 12 receiving twelve seconds. This gives a series of graduated exposure shades on the band, running all the way from white to very dark. The instrument is then carried into the site whose degree of canopy it is desired to measure. The site should be cruised in strips with the photometer held out before the operator, shutter open, and pointing towards the zenith, with the stop watch going. As soon as the operator notes that the exposed band has reached a medium degree of darkness, he closes the shutter which automatically stops the stop-watch. The number of the exposure and the duration is noted down, the paper band turned to the next exposure, the watch re-set, and the operator proceeds on his cruise, repeating the performance until the site has been fully cruised.

The sensitized paper band is removed from the instrument in a dark room and examined under a red light. The colours or shades of the exposures under canopy are matched with those of the first twelve taken in the open and the comparable times noted to produce that particular shade. All the times for exposure under canopy are added up and also the comparable times for exposure in the open. These two total times constitute the essential values in the following equation:

Light intensity under canopy $=$ Time of exposure in open

Light intensity in open $=\frac{\text { Time of exposure under canopy }}{\text { Consder }}$

Consider light intensity in open as 100

Then:

$\begin{aligned} & \text { Light intensity under canopy) } \\ & \text { or light penetration }\end{aligned}=\frac{100 \times \text { Time of exposure in open }}{\text { Time of exposure under canopy }}$

Degree of canopy or shade $=100-$ (degree of light penetration)

For purposes of standard comparison, readings with the Clements Photometer should be taken near noon, under a clear sky, at a season not far removed from the vernal equinox, or about June 21 . Readings taken at 


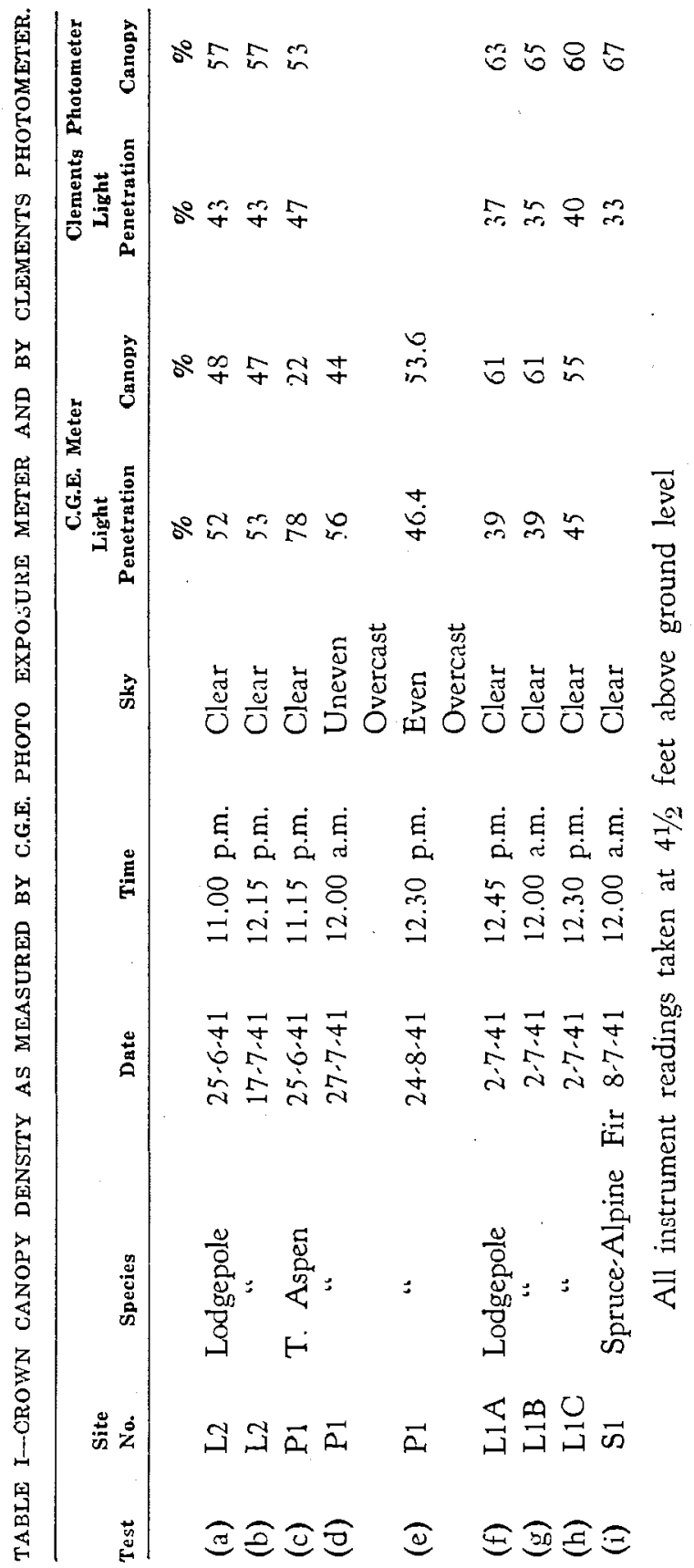


other seasons or at other times of the day may be affected by the different length of shadows cast by the trees.

In 1941 the writer made some tests at the Kananaskis Forest Experiment Station with a C.G.E. Photo Exposure Meter as a means of measuring the degree of forest canopy. The sites were cruised in courses 12 paces apart and readings with the exposure meter were taken every 12 paces on the cruise courses. The normal light intensity in the open was observed with the meter immediately before and after the cruising of each site. The average of the readings under canopy was used with the average reading in the open in the following equation:

Light penetration $=\frac{\text { Average reading under canopy }}{\text { Average reading in open }} \times 100$

Degree of canopy $=100-$ (degree of light penetration) Table I.

The results of canopy measurement by the two methods are shown in

It is apparent that on clear days the Photo Exposure Meter is more affected by sunlight reflected from the tree trunks than is the Clements Photometer. The denser the crown canopy the less direct sunlight strikes the tree trunks to be reflected to the exposure meter so that in dense stands the canopy values obtained with the exposure meter approach more closely those obtained with the photometer, although in every case on a clear day the exposure meter registers more light than does the photometer. (Compare Tests (a) (b) with (f) (g) and (h) in lodgepole pine.)

The effect of sunlight reflected from the more highly reflective trunks of trembling aspen is shown in Test (c). Here the values shown by the exposure meter are so distorted by reflected light as to be almost worthless for standard comparison. In fact in cruising this stand it was noted that on some occasions the readings of the meter showed higher light values than were obtained in the open outside the stand. This led to the inference that better results might be obtained with the exposure meter on a day when the sky was overcast. Some improvement was noted in Test (d) but the overcast was not sufficiently dense or uniform for best results. On one or two occasions during this test the form of the sun could be seen through thinning cloud layers. During Test (e) the sky was uniformly and completely overcast with no individual clouds visible. On this occasion it will be noted that the results obtained with the exposure meter in the aspen stand agreed very closely with the photometer values in (c).

The above tests would appear to indicate that reliable measurements of crown canopy density can be made with a photo-electric exposure meter, provided the readings are made on a day when the sky is uniformly overcast so that the sun is completely covered. It is also probable that under these conditions good results may be obtained over several hours in the middle of the day and that the season of the year is less important than with the photometer. 